\title{
Epidemiological Factors
}

National Cancer Institute

\section{Source}

National Cancer Institute. Epidemiological Factors. NCI Thesaurus. Code C19291.

The identifiable and quantifiable things which contribute to the establishment and transmission of disease. 